\title{
Obesogenic environments: a systematic review of the association between the physical environment and adult weight status, the SPOTLIGHT project
}

\author{
Joreintje D Mackenbach ${ }^{1 *}$, Harry Rutter ${ }^{2}$, Sofie Compernolle ${ }^{3}$, Ketevan Glonti ${ }^{2}$, Jean-Michel Oppert ${ }^{4,5}$,
} Helene Charreire ${ }^{6}$, Ilse De Bourdeaudhuij ${ }^{3}$, Johannes Brug ${ }^{7}$, Giel Nijpels ${ }^{1}$ and Jeroen Lakerveld ${ }^{1}$

\begin{abstract}
Background: Understanding which physical environmental factors affect adult obesity, and how best to influence them, is important for public health and urban planning. Previous attempts to summarise the literature have not systematically assessed the methodological quality of included studies, or accounted for environmental differences between continents or the ways in which environmental characteristics were measured.

Methods: We have conducted an updated review of the scientific literature on associations of physical environmental factors with adult weight status, stratified by continent and mode of measurement, accompanied by a detailed risk-of-bias assessment. Five databases were systematically searched for studies published between 1995 and 2013.

Results: Two factors, urban sprawl and land use mix, were found consistently associated with weight status, although only in North America.

Conclusions: With the exception of urban sprawl and land use mix in the US the results of the current review confirm that the available research does not allow robust identification of ways in which that physical environment influences adult weight status, even after taking into account methodological quality.
\end{abstract}

Keywords: Review, Physical environment, Overweight, Obesity, Adults, Quality assessment

\section{Background}

Obesity prevention is a global public health priority as a result of the worldwide increase in obesity prevalence [1] and its associated chronic diseases [2]. Although genetic factors may underlie the propensity of individuals to become obese [3], the pace at which obesity prevalence has grown at population level during recent decades points to social and environmental causes $[4,5]$. An individual's body mass index (BMI) is mainly determined by energy intake (eating) and energy expenditure (physical activity/sedentary behaviour). These energy

\footnotetext{
* Correspondence: j.mackenbach@vumc.nl

'The EMGO Institute for Health and Care Research, Department of General Practice and Elderly Care Medicine, VU University Medical Center, Amsterdam, The Netherlands

Full list of author information is available at the end of the article
}

balance related behaviours (EBRBs) are influenced by a range of determinants [6]. One important category of determinants is the opportunities for calorie intake and calorie expenditure or a lack thereof in the physical environment. For example, dietary behaviour may be influenced by access to different foods through various types of outlets and services. Similarly, physical activity levels may be influenced by access to recreational or sports facilities, green spaces or parks, as well as transport infrastructure and land use. Certain environments may be more 'obesogenic' than others, such that they are more likely to promote weight gain and obesity in individuals or populations [5], but it remains a challenge to identify the physical environmental factors with the greatest impacts on (the development of) overweight and obesity. 
Following a steady increase in studies relating characteristics of the physical environment to overweight or obesity in the last decade, a number of reviews was published between 2005 and 2012 [7-12], showing mixed results. Among a large range of factors that have been examined, only two environmental correlates appeared to be consistently associated with weight status: indicators of urban sprawl (often based on population density, and positively associated with obesity) and measures of land use mix (negatively associated with obesity) [12,13]. However, there are numerous potential correlates of obesity from the physical environment and it is plausible that many other environmental factors such as access to recreation areas, proximity to fast food outlets or the presence of walking and cycling infrastructure might influence weight status through their links to food and physical activity behavior [14-17]. It may be that the heterogeneity in methods and measures used, or differences in contexts or location, has led to this lack of consistent results.

A recent review of reviews identified a number of gaps and areas for improvement that could explain some of the inconsistencies in the findings about obesogenic environments [12]. The authors evaluated the quality of previous literature reviews on built environment, physical activity and obesity based on: 1) the age and other demographic characteristics of the population, 2) the time frame for the literature search, 3) the total number of articles included, 4) data sources, 5) whether the methodological quality of the primary studies was assessed, 6) whether the measurement mode of the characteristics of the physical environment was reported, 7) whether the outcome was defined, and 8) whether the measurement mode of the outcome was reported. The methodological quality of the primary studies had been assessed in very few of the reviews identified [12]. Although quality assessment tools are rarely used in the evaluation of observational studies [18], assessing the methodological quality of any included studies is an important element of systematic literature reviews.

In addition, previous reviews have generally not distinguished between objective and perceived measures of the environment. Aspects of the physical environment are considered to be measured objectively when assessed through street audits, virtual audits (using remote imaging data) or on the basis of Geographic Information Systems $[19,20]$. The agreement between these objectively measured aspects of physical environments and perceptions of these environments (as measured with interviews or questionnaires) is generally considered to be moderate or low [21,22].

Consequently, we aim to provide an updated review of the literature on physical environmental correlates of adult weight status. We gave a detailed overview of the characteristics of the primary studies and assessed the methodological quality of the included articles. This quality assessment allowed comparison of results of studies that are methodologically strong with those that are methodologically weaker. On the basis that environments are likely to be very different in high income countries from those in middle or low income countries, in this review we have focused on studies that were conducted in high income countries. In addition, we stratified the included studies by mode of measurement and continent of origin.

\section{Methods}

Original studies that examined associations between physical environmental characteristics and adult weight status were reviewed. The physical environment was defined as all built environmental and transport related factors [5]. A literature search, using five electronic databases (PubMed, EMBASE, Web of Science, Cochrane Library and PsychInfo), was conducted in May 2013. Studies relating a physical environmental factor to BMI, overweight or obesity in adults were considered if published between January 1995 and May 2013 in Dutch, English, French or German language. A full description of search terms and search strategy is provided in Additional file 1. Articles were included if weight status in adults was one of the main outcomes. Furthermore, we included articles that focused on macro environmental correlates (i.e. environmental factors measured at the neighbourhood, province or national level) of weight status. Articles of studies were excluded if they:

- focused primarily on socioeconomic characteristics or the social environment of a geographic area

- assessed a physical environmental factor only as a potential confounder

- had a very specific target population that would lead to non-generalizable results (e.g. senior citizens, pregnant women, particular ethnic groups in specific locations, etc.)

- were conducted in low or middle income countries or regions

- did not present original research (e.g. reviews, case reports, editorials, commentaries, discussions or letters)

\section{Data extraction}

The following information was extracted from included studies:

- country (and continent) where the data were collected

- study design

- number of participants

- main environmental determinants 
- whether characteristics of the physical environment were measured objectively or subjectively

- whether weight status was measured or self-reported

- whether the reported associations were or were not in agreement with the hypothesis stated in the article, or whether no association was found

- how the scale of the geographical study area was defined.

The first table presents studies from North America, Australasia, and Europe. Study design was divided into cross-sectional or longitudinal. Physical environmental determinants were categorized into four domains: 1) Physical activity environment, referring to physical activity opportunities, 2) Food environment, referring to food purchasing opportunities, 3) transport opportunities and 4) other (e.g. population density). The definition of the scale of geographical areas was divided into studies that used administrative area limits (such as census tracts or counties), studies that used buffers (for example a $1 \mathrm{~km}$ circular or network buffer around the home) or studies that used a non-standard definition of neighbourhood (for example self-perceived neighbourhood limits).

\section{Assessment of the methodological quality}

For quality assessment, we adapted the Quality Assessment Tool for Quantitative Studies (as developed by the Effective Public Health Practice Project) [23], based on recommendations from a number of authors [24-27]. This contains 19 items in eight key domains for assessment of study quality: 1) study design, 2) blinding, 3) representativeness in the sense of selection bias, 4) representativeness in the sense of withdrawals/drop outs, 5) confounders, 6) data collection, 7) data analysis and 8) reporting and is suitable for assessing observational, as well as experimental studies. Studies can have between six and eight component ratings. An overall rating for each study was determined based on the component ratings, ranging from 1 (low risk-of bias; high methodological quality) to 3 (high risk-of-bias; low methodological quality). For example, if eight ratings were given, strong was attributed to those with no weak ratings and at least five strong ratings, moderate was given to those with one weak rating or fewer than five strong ratings and weak was attributed to those with two or more weak ratings. The quality assessment tool we used is presented in full in Additional file 2.

All included studies were independently assessed for methodological quality by two assessors (JDM and KG). The ratings for each of the eight domains, as well as the total rating, were compared between the two assessors. Consensus was reached on a final rating for each included article.

\section{Results}

After duplicates were removed, 5,642 articles were screened on title and abstract by the first author. Subsequently, a random sample of 500 titles and abstracts was also screened by the second author; 212 full articles were read by the first and second author. Of these articles, 92 were included in the review (see Flow chart in Additional file 3). Characteristics of the studies are shown on Table 1.

A large majority of studies (74) was conducted in North America (USA: 66, Canada: 8), 12 were conducted in Europe (half in the UK) and six were conducted in Australasia. Half the studies (45) were published from 2010 onwards, with seven in 2013. Before 2010, 47 studies were published. Most studies (75) used exclusively objective measures of the physical environment, while 17 studies used perceived measures to link physical environmental characteristics to weight status. Of these 17 studies, nine studies examined both the objective and the perceived environment.

There was great heterogeneity across studies in the use and definition of physical environmental factors. Fifty-three studies investigated the association between an environmental factor that was presumed to affect obesity through physical activity (such as parks or sports facilities), thirty-six studies assessed the association of the food related environment (such as the density of fast-food restaurants) and six studies assessed the transport-related environment (such as proximity to public transport amenities). Thirty-one studies assessed urban form characteristics such as street connectivity, urban sprawl and land use mix, and twenty studies investigated other types of environmental factors such as graffiti or crime. Fifteen studies assessed associations between both food related and physical activity related environments and obesity. It goes beyond the scope of this review to present all the different metrics used, but an overview of the different metrics and associated operationalization of physical environmental factors used in these kind of studies has been provided elsewhere [9]. As an example of the heterogeneity between studies, while fifty-three studies investigated a physical activity related environmental factor, none of the seven $[79,89,104,111,115,116,119]$ studies examining green space used the same definition of this metric. Tilt et al. calculated greenness with NDVI (normalized difference vegetation index; the amount of photosynthetically active light as measured with infrared light) [89], while West et al. defined green space as all publicly owned and operated green spaces [79]. Toftager et al. included beaches, seas, forests and lakes but no agricultural fields [118], while Cummins et al. included agricultural land but excluded domestic gardens [116]. The remaining three studies also used different definitions of green space. 
Table 1 Characteristics of included studies

\begin{tabular}{|c|c|c|c|c|c|c|c|c|c|c|c|c|c|c|c|c|}
\hline \multirow[t]{2}{*}{ First author } & \multirow[t]{2}{*}{ Year } & \multirow[t]{2}{*}{ Country } & \multirow[t]{2}{*}{ Designt } & \multirow[t]{2}{*}{$\mathrm{N}$} & \multicolumn{5}{|c|}{ Domain $^{\ddagger}$} & \multirow[t]{2}{*}{ Main correlates ${ }^{\Omega}$} & \multirow{2}{*}{$\begin{array}{l}\mathrm{O} / \mathrm{P}^{¥} \\
\text { measure } \\
\text { of } \mathrm{PE}\end{array}$} & \multirow{2}{*}{$\begin{array}{l}M / S^{*} \\
\text { weight } \\
\text { status }\end{array}$} & \multicolumn{3}{|c|}{ Association $^{\ddagger}$} & \multirow[t]{2}{*}{ Definition area $^{\S}$} \\
\hline & & & & & $\begin{array}{l}P A \\
\text { envir }\end{array}$ & $\begin{array}{l}\text { Food } \\
\text { envir }\end{array}$ & $\begin{array}{l}\text { Trans- } \\
\text { port }\end{array}$ & $\begin{array}{l}\text { Urban } \\
\text { Form }\end{array}$ & Other & & & & $\begin{array}{l}\text { expec- } \\
\text { ted }\end{array}$ & null & $\begin{array}{l}\text { unex- } \\
\text { pected }\end{array}$ & \\
\hline \multicolumn{17}{|l|}{$\begin{array}{l}\text { Northern } \\
\text { America }\end{array}$} \\
\hline Ahern [28] & 2011 & USA & CS & 3,128 & & $x$ & & & & Restaurants and grocery stores & $\mathrm{O}$ & S & $x$ & & & County \\
\hline Black [29] & 2009 & USA & LG & 48,506 & $x$ & $x$ & & & $x$ & Stores, facilities, crime & $\mathrm{O}$ & S & $x$ & & & Census nbh \\
\hline Black [30] & 2010 & USA & CS & 9,916 & $x$ & $x$ & & & & $\begin{array}{l}\text { Food avail, opportunities \& } \\
\text { barriers to PA }\end{array}$ & $\mathrm{O}$ & S & $x$ & & $x$ & Census nbh \\
\hline Block [31] & 2011 & USA & LG & 3,113 & & $x$ & & & & Proximity to food establishments & O & M & $x$ & $x$ & & Census nbh \\
\hline Bodea [32] & 2009 & USA & CS & 6,893 & $x$ & & & $x$ & & $\begin{array}{l}\text { Street connectivity, residential } \\
\text { density }\end{array}$ & $\mathrm{O}$ & S & $x$ & & & $1 \mathrm{~km}$ buffer \\
\hline Bodor [33] & 2010 & USA & CS & 3,925 & $x$ & & & & & Food environment & $\mathrm{O}$ & s & $x$ & $x$ & & $2 \mathrm{~km}$ buffer \\
\hline Brown [34] & 2009 & USA & CS & 5,000 & $x$ & & $x$ & $x$ & & Walkable land use, destinations & $\mathrm{O}$ & S & $x$ & $x$ & $x$ & Census block group \\
\hline Brown [35] & 2013 & USA & $\mathrm{CS}$ & 3.528 & $x$ & & & & & Walkability, bikeability & $\mathrm{O}$ & M & $x$ & & & Census block group \\
\hline Casagrande [36] & 2011 & USA & CS & 3,493 & $x$ & & & & & Walkability & $\mathrm{O}$ & M & & $x$ & & Census nbh \\
\hline Chen [37] & 2012 & USA & $\mathrm{CS}$ & 3,550 & & $x$ & & & & Food outlets & $\mathrm{O}$ & S & $x$ & $x$ & & 0.5 mile buffer \\
\hline Doyle [38] & 2006 & USA & CS & 9,252 & $x$ & & & & $x$ & Walkability, crime & $\mathrm{O}$ & M & $x$ & & & County \\
\hline Drewnowski [39] & 2012 & USA & CS & 1,682 & & $x$ & & & & $\begin{array}{l}\text { Proximity and price of } \\
\text { supermarkets }\end{array}$ & $\mathrm{O}$ & S & & & $x$ & $\begin{array}{l}1 \text { mile distance to } \\
\text { supermarket }\end{array}$ \\
\hline Eid [40] & 2008 & USA & $\mathrm{CS}$ & 5,500 & & & & $x$ & & Urban sprawl & $\mathrm{O}$ & $S$ & & $x$ & & 2 mile radius disc \\
\hline Ewing [41] & 2003 & USA & $\mathrm{CS}$ & 206,992 & & & & $x$ & & Urban sprawl & $\mathrm{O}$ & S & $x$ & & & County \\
\hline Frank [42] & 2004 & USA & $\mathrm{CS}$ & 10,878 & $x$ & & & $x$ & & Land use mix & O & S & $x$ & & & $\begin{array}{l}\text { Block group/square } \\
\text { kilometer }\end{array}$ \\
\hline Frank [43] & 2008 & USA & CS & 13,065 & & & & $x$ & & $\begin{array}{l}\text { Residential density, street } \\
\text { connectivity, land use mix }\end{array}$ & $\mathrm{O}$ & S & $x$ & & & $1 \mathrm{~km}$ distance \\
\hline Frank [44] & 2007 & USA & CS & 2,056 & $x$ & & & & & Walkability & O & $S$ & $x$ & & & $\begin{array}{l}\text { Road polygons } \\
\text { traveling } 1 \mathrm{~km} \text { from } \\
\text { house }\end{array}$ \\
\hline Frank [45] & 2007 & USA & CS & 1,228 & $x$ & & & & & Walkability & $\mathrm{O}$ & S & $x$ & & & $1 \mathrm{~km}$ network buffer \\
\hline Frank [46] & 2009 & USA & $\mathrm{CS}$ & & & $x$ & & & & Food outlet visits, walkability & $\mathrm{O}$ & $S$ & $x$ & $x$ & & $1 \mathrm{~km}$ \\
\hline Gibson [47] & 2011 & USA & LG & 8,100 & & $x$ & & & & Food environment & $\mathrm{O}$ & S & $x$ & & & ZIP code area \\
\hline Gregson [48] & 2011 & USA & CS & 14,205 & & $x$ & & $x$ & & Sprawl and restaurant types & $\mathrm{O}$ & $S$ & $x$ & & $x$ & County \\
\hline Hattori [49] & 2013 & USA & $\mathrm{CS}$ & 97,678 & & $x$ & & & & nbh food outlets & $\mathrm{O}$ & S & & $x$ & & $\begin{array}{l}1 \text { mile Euclidian } \\
\text { distance }\end{array}$ \\
\hline Hoehner [50] & 2011 & USA & CS & 17,000 & $x$ & & & & & Walkability & O & M & $x$ & & & Block group \\
\hline
\end{tabular}


Table 1 Characteristics of included studies (Continued)

\begin{tabular}{|c|c|c|c|c|c|c|c|c|c|c|c|c|c|c|c|c|}
\hline Hutchinson [51] & 2012 & USA & CS & 1,243 & & $x$ & & & & $\begin{array}{l}\text { Availability of healthy \& unhealthy } \\
\text { foods }\end{array}$ & $\mathrm{O}$ & $\mathrm{S}$ & $x$ & $x$ & & Census tract \\
\hline Inagami [52] & 2009 & USA & CS & 2,156 & & $x$ & & & & $\begin{array}{l}\text { Fast food \& restaurant } \\
\text { concentration }\end{array}$ & $\mathrm{O}$ & $\mathrm{S}$ & $x$ & & & Census tract \\
\hline James [53] & 2012 & USA & CS & 68,000 & & & & $x$ & & County sprawl index & $\mathrm{O}$ & $\mathrm{S}$ & $x$ & & & County \\
\hline Jeffery [54] & 2006 & USA & CS & 1,033 & & $x$ & & & & Proximity of (fastfood) restaurants & $\mathrm{O}$ & $S$ & & $x$ & & $\begin{array}{l}0.5 \text { miles, } 1 \text { miles and } \\
2 \text { miles from home } \\
\text { and work adresses }\end{array}$ \\
\hline Jilcott [55] & 2010 & USA & CS & 9,800 & & $x$ & & & & Food retail gap & $\mathrm{O}$ & $S$ & $x$ & & & County \\
\hline Keegan [56] & 2012 & USA & CS & 133,000 & & & & $x$ & $x$ & $\begin{array}{l}\text { Population \& housing density, } \\
\text { commuting characteristics }\end{array}$ & $\mathrm{O}$ & $S$ & & $x$ & & Census nbh \\
\hline Lesser [57] & 2013 & USA & CS & 2,589 & & $x$ & & & & Outdoor food advertising & $\mathrm{O}$ & $\mathrm{S}$ & $x$ & & & Census tract \\
\hline Lopez [58] & 2004 & USA & CS & 104,084 & & & & $x$ & & Urban sprawl & $\mathrm{O}$ & $S$ & $x$ & & & $\begin{array}{l}\text { Tracts of approx } 4000 \\
\text { people }\end{array}$ \\
\hline Lopez [59] & 2007 & USA & CS & 15,358 & $x$ & $x$ & & $x$ & $x$ & Various & $\mathrm{O}$ & $S$ & $x$ & $x$ & & ZCTA \\
\hline Lovasi [60] & 2009 & USA & CS & 13,102 & $x$ & & & & & Walkability & $\mathrm{O}$ & M & $x$ & & & $1 \mathrm{~km}$ buffer \\
\hline Lovasi [61] & 2012 & USA & CS & 13,102 & $x$ & & $x$ & & $x$ & Walkability, aesthetics, safety & $\mathrm{O}$ & M & $x$ & $x$ & $x$ & $1 \mathrm{~km}$ buffer \\
\hline McDonald [62] & 2012 & USA & CS & 690 & $x$ & & & & & Walkability & $\mathrm{O}$ & M & & $x$ & & Census block group \\
\hline Mehta [63] & 2008 & USA & CS & 714,054 & & $x$ & & & & Restaurant mix & $\mathrm{O}$ & $S$ & $x$ & & & County \\
\hline Mobley [64] & 2006 & USA & CS & 2,692 & $x$ & $x$ & & $x$ & $x$ & $\begin{array}{l}\text { Fitness facilities, food establish- } \\
\text { ments, crime }\end{array}$ & $\mathrm{O}$ & & $x$ & $x$ & & ZIP code areas \\
\hline Morland [65] & 2006 & USA & CS & 10,763 & & $x$ & & & & Food stores & $\mathrm{O}$ & & $x$ & & & Census tract \\
\hline Oka [66] & 2012 & USA & CS & 5,485 & $x$ & $x$ & $x$ & & & Various & $\mathrm{O}$ & M & & $x$ & & Census tract \\
\hline Plantinga [67] & 2007 & USA & LG & 4,700 & & & & $x$ & & Sprawl & $\mathrm{O}$ & $S$ & $x$ & & & County \\
\hline Plantinga [68] & 2007 & USA & CS & 3,607 & & & & $x$ & & Sprawl & $\mathrm{O}$ & $S$ & & $x$ & & County \\
\hline Rundle [69] & 2007 & USA & CS & 13,102 & $x$ & & $x$ & $x$ & & $\begin{array}{l}\text { Land use, bus stop density, pop } \\
\text { density, intersection density }\end{array}$ & $\mathrm{O}$ & M & $x$ & $x$ & & Census tract area \\
\hline Rundle [70] & 2008 & USA & CS & 13,102 & $x$ & $x$ & & & & $\begin{array}{l}\text { Density of BMI-healthy food out- } \\
\text { lets and walkability }\end{array}$ & $\mathrm{O}$ & M & $x$ & $x$ & & $\begin{array}{l}805 \text { meter network } \\
\text { buffer }\end{array}$ \\
\hline Rundle [71] & 2009 & USA & CS & 13,102 & $x$ & $x$ & & & & Food environment and walkability & $\mathrm{O}$ & M & $x$ & $x$ & & $\begin{array}{l}\text { Half-mile radius } \\
\text { circular buffers }\end{array}$ \\
\hline Rundle [72] & 2013 & USA & CS & 13,102 & $x$ & & & & & Park characteristics & $\mathrm{O}$ & M & $x$ & $x$ & & $\begin{array}{l}\text { Half-mile radius } \\
\text { circular buffers }\end{array}$ \\
\hline Sallis [73] & 2009 & USA & CS & 2,199 & $x$ & & & & & Walkability & $\mathrm{O}$ & $\mathrm{S}$ & $x$ & & & Block groups \\
\hline Samimi [74] & 2009 & USA & CS & 300,000 & & & $x$ & $x$ & & $\begin{array}{l}\text { Transport, land use, built } \\
\text { environment }\end{array}$ & $\mathrm{O}$ & $S$ & $x$ & & & Census tract \\
\hline Scott [75] & 2009 & USA & CS & 1,750 & $x$ & & & $x$ & $x$ & $\begin{array}{l}\text { Safety, des- tina- tions, social fac- } \\
\text { tors }\end{array}$ & $\mathrm{O}$ & $S$ & $x$ & $x$ & & Urban census tract \\
\hline
\end{tabular}


Table 1 Characteristics of included studies (Continued)

\begin{tabular}{|c|c|c|c|c|c|c|c|c|c|c|c|c|c|c|c|c|}
\hline Smith [76] & 2008 & USA & CS & 453,927 & $x$ & & & & & Walkability & $\mathrm{O}$ & $S$ & $x$ & $x$ & & Census block group \\
\hline Smith [77] & 2011 & USA & CS & 100,000 & $x$ & & & & & Walkability & O & $S$ & $x$ & $x$ & & Census block group \\
\hline Wang [78] & 2007 & USA & CS & 7,595 & & $x$ & & & & $\begin{array}{l}\text { Proximity \& density of fastfood } \\
\text { restaurants \& food retail }\end{array}$ & $\mathrm{O}$ & $S$ & $x$ & $x$ & & $\begin{array}{l}\text { Combination of } \\
\text { census tracks and } \\
\text { block groups }\end{array}$ \\
\hline West [79] & 2012 & USA & CS & 99,534 & $x$ & & & & & Park(land) area & $\mathrm{O}$ & S & $x$ & & & $\begin{array}{l}\text { metropolitan statistical } \\
\text { area }\end{array}$ \\
\hline Yamada [80] & 2011 & USA & CS & 4,960 & & & & $x$ & & mixed land use & O & $S$ & $x$ & & & $\begin{array}{l}\text { Block group, tract, } \\
1 \mathrm{~km} \text { buffer }\end{array}$ \\
\hline Zhao [81] & 2010 & USA & LG & & & & & $x$ & & Urban sprawl & O & $S$ & $x$ & & & $\begin{array}{l}\text { Census metropolitan } \\
\text { areas/county level }\end{array}$ \\
\hline Zick [82] & 2009 & USA & CS & 453,927 & $x$ & $x$ & & & & Food and PA opportunities & $\mathrm{O}$ & S & $x$ & $x$ & & Block groups \\
\hline Zick [83] & 2013 & USA & CS & 35,685 & $x$ & & & & & Walkability & O & $S$ & $x$ & & & Census block \\
\hline Bai [84] & 2013 & USA & CS & 893 & $x$ & & & & & Park quality & P & $S$ & $x$ & $x$ & & $\begin{array}{l}\text { Census block within } \\
0.5 \text { miles of park }\end{array}$ \\
\hline Catlin [85] & 2003 & USA & CS & 2,821 & $x$ & $x$ & & & & $\begin{array}{l}\text { Community perceptions, } \\
\text { community infrastructure, PA } \\
\text { facilities }\end{array}$ & P & S & $x$ & $x$ & & Community \\
\hline Mujahid [86] & 2008 & USA & CS & 2,865 & $x$ & $x$ & & & $x$ & Neighborhood conditions & $P$ & M & $x$ & $x$ & & Census tract \\
\hline $\begin{array}{l}\text { Powell-Wiley } \\
\text { [87] }\end{array}$ & 2013 & USA & CS & 5,907 & $x$ & $x$ & & & $x$ & $\begin{array}{l}\text { nbh violence, physical } \\
\text { environment, social cohesion }\end{array}$ & $P$ & M & $x$ & $x$ & & Neighborhood \\
\hline Wilson [88] & 2007 & USA & CS & 1,111 & $x$ & & & & $x$ & $\begin{array}{l}\text { opportunities for PA and pleasant } \\
\text { neighbourhoods }\end{array}$ & $P$ & $S$ & $x$ & $x$ & & $\begin{array}{l}\text { Neighborhood and } \\
\text { community }\end{array}$ \\
\hline Tilt [89] & 2007 & USA & CS & 529 & $x$ & & & & & greenness, accessibility & O\&P & S & $x$ & $x$ & & 0.4 mile distance \\
\hline Boehmer [90] & 2007 & USA & CS & 1,032 & $x$ & & $x$ & $x$ & $x$ & $\begin{array}{l}\text { Facilities land use, transportation, } \\
\text { aesthetics }\end{array}$ & O\&P & $S$ & $x$ & $x$ & $x$ & $400 \mathrm{~m}$ radius \\
\hline Rutt [91] & 2005 & USA & CS & 996 & $x$ & & & $x$ & $x$ & Various & O\&P & $S$ & & $x$ & & $\begin{array}{l}1 / 4 \text { mile radius and } \\
2.5 \text { mile radius }\end{array}$ \\
\hline Pendola [92] & 2007 & USA & CS & 670 & & & & $x$ & $x$ & $\begin{array}{l}\text { Population density, sense of } \\
\text { community }\end{array}$ & O\&P & $S$ & & $x$ & & Census tract \\
\hline Joshu [93] & 2008 & USA & CS & 1,818 & & & & $x$ & & Sprawl, urbanization & O\&P & $S$ & $x$ & & & FIPS code \\
\hline Berry [94] & 2010 & Canada & $C S \& L G$ & $\begin{array}{l}572 \\
\text { and } \\
1,164\end{array}$ & $x$ & & & & & $\begin{array}{l}\text { Ease of walking and proximity to } \\
\text { outdoor recreation }\end{array}$ & O & $S$ & & $x$ & & Census nbh \\
\hline Spence [95] & 2009 & Canada & CS & 2,900 & & $x$ & & & & $\begin{array}{l}\text { RFEI (retail food environment } \\
\text { index) }\end{array}$ & O & S & $x$ & $x$ & & $800 \mathrm{~m}$ and $1600 \mathrm{~m}$ \\
\hline Pouliou [96] & 2010 & Canada & CS & 115,548 & $x$ & $x$ & & $x$ & & Built environment & $\mathrm{O}$ & $S$ & $x$ & & & Subprovincial scale \\
\hline Prince [97] & 2012 & Canada & CS & 6,564 & $x$ & $x$ & & & $x$ & $\begin{array}{l}\text { Recreation, social, food } \\
\text { environment }\end{array}$ & $\mathrm{O}$ & $S$ & $x$ & $x$ & & $\begin{array}{l}\text { Neighborhoods based } \\
\text { on natural barriers? }\end{array}$ \\
\hline
\end{tabular}


Table 1 Characteristics of included studies (Continued)

\begin{tabular}{|c|c|c|c|c|c|c|c|c|c|c|c|c|c|c|c|}
\hline Prince [98] & 2011 & Canada & CS & 5,025 & $x$ & $x$ & & $x$ & $\begin{array}{l}\text { Recreation, social, food } \\
\text { environment }\end{array}$ & $\mathrm{O}$ & $S$ & $x$ & $x$ & & $\begin{array}{l}\text { Neighborhoods based } \\
\text { on natural barriers? }\end{array}$ \\
\hline Ross [99] & 2007 & Canada & CS & 33,000 & & & $x$ & $x$ & $\begin{array}{l}\text { Dwelling density, sprawl, } \\
\text { immigration }\end{array}$ & $\mathrm{O}$ & S & $x$ & $x$ & & Census tract area \\
\hline Kestens [100] & 2012 & Canada & CS & 5,578 & & $x$ & & & Food environment & $\mathrm{O}$ & S & $x$ & $x$ & & Census nbh \\
\hline Berry [101] & 2010 & Canada & $C S \& L G$ & 500 & $x$ & & & & Perceptions of neighbourhood & O\&P & $S$ & & $x$ & & Census nbh \\
\hline \multicolumn{16}{|l|}{ Australasia } \\
\hline Garden [102] & 2008 & Australia & CS & 7,290 & & & $x$ & & Urban sprawl & O & S & $x$ & & & $\begin{array}{l}\text { Local government } \\
\text { area }\end{array}$ \\
\hline Pearce [103] & 2009 & $\begin{array}{l}\text { New } \\
\text { Zealand }\end{array}$ & CS & 12,529 & & $x$ & & & distance to fastfood outlet & O & M & & $x$ & $x$ & $\begin{array}{l}\text { meshblock } \\
\text { neighbourhoods }\end{array}$ \\
\hline Richardson [104] & 2013 & $\begin{array}{l}\text { New } \\
\text { Zealand }\end{array}$ & CS & 8,157 & $x$ & & & & Urban green space & $\mathrm{O}$ & M & & $x$ & & Census area unit \\
\hline Christian [105] & 2011 & Australia & CS & 1,151 & $x$ & $x$ & & & Built \& social environment & O\&P & $S$ & $x$ & $x$ & & $\begin{array}{l}1.6 \mathrm{~km} \text { road network } \\
\text { service area }\end{array}$ \\
\hline Gebel [106] & 2011 & Australia & $L G$ & 1,027 & $x$ & & $x$ & & $\begin{array}{l}\text { Walkability, dwelling density, land } \\
\text { use mix }\end{array}$ & O\&P & $S$ & $x$ & $x$ & & $\begin{array}{l}\text { Census collector } \\
\text { district }\end{array}$ \\
\hline Giles-Corti [107] & 2003 & Australia & CS & 1,803 & $x$ & & & & Physical environment & O\&P & $\mathrm{S}$ & $x$ & & & Collector districts \\
\hline \multicolumn{16}{|l|}{ Europe } \\
\hline Ball [108] & 2012 & UK & CS & 1,062 & $x$ & & & & Street connectivity & O & S & & & $x$ & Datazone \\
\hline Van Dyck [109] & 2010 & Belgium & CS & 1,200 & $x$ & & & & Walkability & $\mathrm{O}$ & S & & $x$ & & Statistical sectors \\
\hline Santana [110] & 2009 & Portugal & CS & 7,669 & $x$ & $x$ & $x$ & $x$ & $\begin{array}{l}\text { Environmental disadvantages \& } \\
\text { opportunities }\end{array}$ & O & $S$ & $x$ & $x$ & & Neighbourhood? \\
\hline Ellaway [111] & 2005 & Europe & CS & 6,919 & $x$ & & & $x$ & Graffiti, greenery & $\mathrm{O}$ & $S$ & $x$ & & & $\begin{array}{l}\text { immediate residential } \\
\text { environment }\end{array}$ \\
\hline Macdonald [112] & 2011 & UK & CS & 991 & & $x$ & & & Distance to food stores & O & $S$ & & $x$ & $x$ & 500 and 1000 meter \\
\hline Leal [113] & 2012 & France & CS & 7,230 & & & $x$ & $x$ & $\begin{array}{l}\text { Sociodemographic factors, } \\
\text { physical factors, service-related, } \\
\text { social-interactional environment }\end{array}$ & O & M & $x$ & $x$ & & $\begin{array}{l}500 \mathrm{~m} \text { radius/TRIRIS } \\
\text { geographic unit }\end{array}$ \\
\hline Burgoine [114] & 2011 & UK & CS & 893 & & $x$ & $x$ & & $\begin{array}{l}\text { Residential density, street } \\
\text { connectivity, land use mix }\end{array}$ & O & S & $x$ & $x$ & & LSOA/MSOA \\
\hline Coombes [115] & 2010 & UK & CS & 6,821 & $x$ & & & & Green space & $\mathrm{O}$ & $S$ & $x$ & & & $\begin{array}{l}\text { Not applicable } \\
\text { (distance)/800 meter }\end{array}$ \\
\hline Cummins [116] & 2012 & UK & CS & 79,136 & $x$ & & & & Percentage greenspace & $\mathrm{O}$ & $S$ & & $x$ & $x$ & MSOA \\
\hline Poortinga [117] & 2006 & UK & CS & 14,836 & & & $x$ & $x$ & $\begin{array}{l}\text { Friendliness, trust, social capital, } \\
\text { access }\end{array}$ & $P$ & M & $x$ & $x$ & & Postcode sectors \\
\hline
\end{tabular}


Table 1 Characteristics of included studies (Continued)

\begin{tabular}{|c|c|c|c|c|c|c|c|c|c|c|}
\hline Toftager [118] & 2011 & Denmark & CS & 21,832 & $x$ & Distance to green space & $P$ & $S$ & $x$ & $\begin{array}{l}\text { Not applicable } \\
\text { (distance) }\end{array}$ \\
\hline Nielsen [119] & 2007 & Denmark & CS & 2,000 & $x$ & $\begin{array}{l}\text { Perceptions of distance to } \\
\text { garden/green area }\end{array}$ & $P$ & $S$ & $x$ & $\begin{array}{l}\text { Not applicable } \\
\text { (distance) }\end{array}$ \\
\hline
\end{tabular}

†CS = cross-sectional, $L G=$ longitudinal.

$\neq \mathrm{x}=$ association was statistically significant in this direction.

$¥$ Measures of the Physical Environment $(P E)$ : $O=$ objective, $P=$ perceived. Measures of weight status: $M=$ objectively measured, $S=$ self-reported.

$\Omega \mathrm{PA}=$ physical activity, $\mathrm{nbh}=$ neighbourhood.

$\S \mathrm{nbh}=$ neighbourhood, $\mathrm{km}=$ kilometer, $\mathrm{m}=$ meter, $\mathrm{LSOA} / \mathrm{MSOA}=\mathrm{lower} /$ middle statistical output area, $\mathrm{ZCTA}=$ Zip Code Tabulation Area. 
There was no consistent pattern of associations between physical environmental factors and weight status. For example, nineteen studies assessed the association between walkability (often a combination of three factors: intersection density, land use mix and population density) and overweight or obesity - one study from Europe and 18 studies from North America. The European study did not find an association between walkability and obesity, although there was an association between walkability and several domains of physical activity [109]. Of the other 18 studies, eight found associations that were in line with the hypothesis (i.e. higher walkable areas are associated with lower BMI or overweight prevalence). Three studies did not find statistically significant associations and five studies found inconsistent results. Inconsistencies arose from results that indicated that associations were only present in men versus women, only present in disadvantaged areas versus higher SES neighbourhoods, or only present for BMI versus overweight/obesity as outcome.

Only eight studies used longitudinal data. The followup time ranged from four to 25 years. Sixty-six studies defined the geographical scale of study ('neighbourhood' or 'environment') based on administrative boundaries (for example: county or census tract). The remaining studies used 'buffers' (network or Euclidian) with varying radius, with the exception of one study that used limits of activity-space. Of the 93 included studies, 36 presented results that broadly corresponded with the hypothesis in the study (i.e. the results were according to what was expected). Fourteen studies reported that they did not find statistically significant results and 5 studies reported unexpected results, i.e. opposite to the hypothesised direction. Another 38 studies reported on a mixture of expected, unexpected and/or non-significant results.

\section{Intercontinental differences}

Two environmental measures were relatively consistently and statistically significantly associated with overweight status or BMI; urban sprawl and land use mix. Urban sprawl was studied in twelve studies (none of the European studies, 1 Australian study and 11 North American studies): eight reported on significant associations in the expected direction (more urban sprawl was related to more obesity), and four reported no association. Land use mix (separately from walkability) was examined in five North American studies, and was significantly associated with obesity in all of these studies (less land use mix was related to more obesity). One study from the UK showed that land use mix was not significantly associated with overweight or obesity [114]. However, because of the dominance of North American studies, it was not possible to differentiate between studies from European, Australasian or North American origin.
In Europe, five $[111,115,116,118,119]$ out of twelve studies investigated the role of green space in the risk for overweight or obesity. Although Nielsen \& Hansen, Toftager and Ellaway et al. found results supporting the hypothesis that green space is associated with lower BMI $[111,118,119]$, the results of Cummins \& Fagg and Coombes et al. did not support this hypothesis $[115,116]$. Other European studies did not provide clear evidence about any physical environmental factors associated with overweight or obesity.

It was not possible to conclude which physical environmental factors were specific to Australasia with regards to the relation between characteristics of the physical environment and obesity. The two studies from New Zealand examined fast-food outlets [103] and green space [104] (no significant associations) and the four studies from Australia examined several physical environmental factors such as type of street, spatial access to natural facilities, graffiti and street connectivity.

\section{Objective versus perceived measures}

Six studies used only perceived measures of the environment (for example; perceived distance to green space or perceptions of access to amenities), but the range of factors studied was broad: in total, over 20 different factors were examined in these six studies. Nine studies assessed both perceived and objectively measured environment, but most studies did not assess the same factors objectively as subjectively. Tilt et al. and Boehmer et al. were the only authors who were able to compare the objective and subjective measures. Tilt et al. concluded that only objectively measured factors (accessibility and greenness) were associated with BMI [89], while Boehmer et al. concluded that perceived as well as objective measures of land use and aesthetics were the most robust correlates of obesity, compared to a range of other factors [90]. Gebel et al. used an alternative approach by showing that a mismatch between objectively measured and perceived walkability measures was associated with weight gain: those who perceived a highly walkable area as being of low walkability showed a larger increase in BMI than those with concordant perceptions [106].

\section{Methodological quality assessment}

Overall, for 29 articles the methodological quality was rated as strong, for 53 articles as moderate and for 8 as weak (full details on the quality assessment are provided in Additional file 4). Eleven articles were rated as weak regarding representativeness, and 45 and 31 were rated as strong and moderate respectively regarding representativeness (five articles did not receive a rating because they referred to a design article but did not provide any other information [35,72,82,100,104]). Forty-six articles scored weak on data collection as they did not provide 
information about the validity or reliability of their measures, twenty-seven scored moderate and nineteen articles scored strong. In terms of confounding five papers were rated as moderate and one as weak as these studies did not adjust for income. All studies except one scored strong on 'analysis'. Seven studies scored moderate on reporting; all other studies were rated as strong on this issue. There appeared to be no association between the overall methodological quality of the reviewed article and the likelihood of reporting associations that were in line with the authors' hypotheses. Of the 11 studies that received an overall weak score, three studies solely reported results that were in concordance with the hypothesis. Of the moderate (53) and strong (30) studies, 19 and 10 reported results that were in concordance with the hypothesis, respectively.

\section{Discussion}

We systematically reviewed the published scientific literature on associations between physical environmental factors and weight status in adults. The results showed a great heterogeneity in findings. In line with previous results $[4,7,9,10,12]$, two environmental variables appeared to be more consistently associated with overweight or obesity than other factors: 'urban sprawl' and 'land use mix'. Of note, these two factors have been widely studied in North America, but not in Europe or Australasia. For other environmental variables, there was great variation in the metrics used, the number of features studied and the different contexts of the studies. The current evidence base therefore provides inconsistent results about associations between the physical environment and overweight or obesity in adults.

Previous literature reviews have generally shown a positive association between the physical (or built) environment and obesity. For example, Booth et al. concluded that there was 'strong preliminary evidence of a relationship between built environment features and the prevalence of obesity'. However, the authors only included 9 papers [4]. Papas et al. included 20 studies, and stated that ' 17 found a statistically significant relation between some aspect of the built environment and risk of obesity' [7]. Feng et al. included 63 studies, and were cautious in drawing their conclusions: 'While there is strong intuitive appeal to the notion that the built environment must be contributing to the obesity epidemic, existing scientific evidence does not provide consistent or convincing support for this hypothesis' [9].

We hypothesized that this lack of apparent inconsistent results was due to heterogeneity in the measures and methods used in primary studies. We therefore conducted stratified analyses, grouping homogeneous studies together in order to identify any patterns that might emerge if we reduced heterogeneity. Although some authors stated that there was limited generalizability of North American results to European settings [7,116], findings remained heterogeneous. As there were not enough studies taking into account similar physical environmental factors between North America, Australia and Europe, it was not possible to make intercontinental comparisons. It may well be that in developed societies, the differences between physical environments within one country or city are not large enough to create measurable differences in impact on outcomes.

Stratification by mode of measurement did not reveal consistent differences between studies that used objective measures versus perceptions of the environment. Although a number of tools to measure perceptions of the environment are available (for example: NEWS [120], ALPHA [121], NWS [122], PANES [123]), many authors used a self-developed instrument, making it difficult to compare results between studies. Comparison of studies that used both objective measures and perceptions of the environment was hampered by to the fact that different dimensions are assessed with these measurement modes. While researchers often objectively assessed distance to a specific facility, they tended to ask for general perceptions of distance: for example is this facility present in your neighbourhood' instead of 'do you think this facility is present within 2.5 kilometres. It was noteworthy that no studies reporting on perceptions of the environment found associations that were contrary to the author's prior hypotheses. This might indicate that unexpected associations were not found, that unexpected associations were not reported (or published), or that prior hypotheses were not tightly defined.

Taking into account the methodological quality of the primary studies did not lead to different results from previous reviews. This may, however, be a function of the approach taken by the tool: it is possible that the determining factor is not the quality of the study, but rather the conceptual model it is based on. As Ding and Gebel [12] describe, we should look for more complex conceptual and statistical models, taking into account innovative analyses and distinguishing between objective and subjective measures of the environment. A relatively simple quality assessment may not be capable of discerning the factors that differentiate these more sophisticated approaches.

Although there is a general consensus that the physical environment has an important influence on individuals' weight status (in environments where there is no food, one cannot eat; in environments where there are no cars, public transport or machines, one cannot avoid being more physically active for transport, daily activities or work), a large body of research has failed robustly to identify direct causal pathways between the physical environment and weight status. We found no evidence that 
continent, mode of measurement of the physical environmental correlates, or the methodological quality of primary studies affected the consistency of the results. There may, however, be a number of additional explanations for this lack of consistent associations.

An extra set of quality criteria - specific for studies relating environmental factors to health or weight status could therefore be defined. This could include taking into account interactions of objective measures and perceptions, the effect of mediators and moderators, and the complexity of conceptual models. Crucial mediators obviously include energy balance related behaviours (EBRBs) such as dietary habits and levels of physical activity, and future studies would benefit from including these kinds of intermediate outcomes. While physical activity mediators have been examined in some studies $[36,45,117,118]$, only two of the reviewed studies took into account food related mediators [86,113]. Furthermore, different environmental variables may moderate each other's influences or may be moderated by individual level determinants. For example, people with high self- efficacy for physical activity or who perceive strong social support or social pressure to be physical active, may be less influenced by physical environments that do not support physical activity [124] than those with lower levels of such factors. A number of studies that have recently explored mediation and moderation between individual level and family and neighbourhood environmental level determinants have been conducted recently, and indeed suggest such relationships in their associations with EBRB and weight status (i.e. [125]. It may also be necessary to more critically assess the methods used for assessing objective and subjective measures of the environment. Even objective measures are only able to capture part of the 'true' physical environment, and perceptions of the environment may be heavily influenced by demographic or lifestyle factors.

Then, it may be that the areas in which the included studies were conducted do not provide adequate variety in exposure to result in measurable differences in outcomes. Indeed, many studies were conducted in only a single city or region. It may be valuable to assess physical environmental factors in a wider region (for example; the approach taken by the SPOTLIGHT project [126]). Additionally, the use of administrative units may be ill-suited to examine environmental effects on health. The impact of exposure to environmental variables in a neighbourhood, area or place may differ between individuals $[127,128]$, so previous studies might have misclassified relevant study areas. Furthermore, it would advance the field if more emphasis were placed on the difference between causation and correlation. Longitudinal observational studies and natural experiments have the advantage of allowing for temporal associations, while accounting for residential self-selection (endogeneity) [73] may also be possible in cross-sectional study designs.

Future researchers should consider the complexity of the relations with individual weight status, as simplistic interventions aimed at limited aspects of the physical environment may not provide the desired changes in obesity-related behaviours, let alone outcomes such as weight status. There is, for example, the potential for compensatory behaviours: more active people might consume more food, or people who use active transport may reduce physical activity in other domains of their lives. It needs to be understood which health-related activities people conduct where, when, for how long, with whom and so on, and also to include thorough appraisal of the different tools that measure perceptions of the environment in terms of validity, reliability and applicability.

\section{Strengths and limitations}

Strengths of the present review are the adherence to essential criteria for literature reviews suggested by Ding and Gebel [12], the systematic assessment of methodological quality of the primary studies and the inclusion of articles that have been published since previous reviews. In addition, we stratified the results by continent and mode of measurement. However, there are also a number of limitations to this systematic literature review. We aimed to improve the methodological quality of the systematic literature review, but the quality assessment tool posed a number of challenges. First, it was difficult to assess the representativeness of the study samples. There is no consensus as to whether one should judge the representativeness on the response rate, on the sample size or on the characteristics of the sample. The assessment of the representativeness of the sample can be context-specific. Second, some papers did not present all necessary information, but instead referred to a design article published elsewhere. As the current review assessed the quality of the reviewed studies based on what was reported in the original publications - and it may be that not all relevant information regarding the quality criteria was reported - lower scores on the quality assessment may not necessarily reflect a low quality of the study but might merely have been a lack of reported detail in the paper. Third, the comparison between strong, moderate and weak articles in terms of finding results consistent with the hypothesis may have been hindered by publication or reporting bias.

Finally, by excluding articles that assessed physical environmental factors as potential mediators only, the selection of articles included in this review may be biased towards positive findings. Indeed, authors may respond to null or unexpected findings by changing the emphasis of their manuscript (for example using the physical environmental factor as confounder only). As a result, the 
inclusion of articles that treated physical environmental factors as confounders would be likely to strengthen our conclusion that the overall evidence for an association between environmental factors and weight status is weak.

\section{Conclusions}

We systematically assessed the methodological quality of the included studies and took this quality into account in the review and interpretation of the evidence. The results of the present review remain in line with previous literature reviews $[4,7,9,12]$, indicating that this additional step did not lead to different conclusions.

This systematic review provides an updated overview of the studies examining associations between the physical environment and weight status. We add to the existing literature by stratifying articles by continent and mode of measurement. The fact that this extensive review showed minimal evidence for an association between characteristics of the built environment and weight status indicates that we still do not fully understand the complex relations involved.

Although land use mix and urban sprawl were more consistently associated with overweight or obesity than other physical environmental factors, the evidence remains weak and the nature of associations between the physical environment and weight status needs further study.

\section{Additional files}

\section{Additional file 1: Search strategy. \\ Additional file 2: Quality Assessment Tool. \\ Additional file 3: Flowchart. \\ Additional file 4: Quality assessment of the included studies.}

\section{Abbreviations}

BMI: Body mass index; EBRB: Energy balance related behaviour.

\section{Competing interests}

The authors declare that they have no competing interest.

\section{Authors' contributions}

JDM carried out the literature search, performed the quality assessment of included articles and drafted the manuscript. HR and $J \mathrm{~L}$ helped draft the manuscript. KG performed the quality assessment as second reviewer. All authors commented on and approved the final manuscript

\section{Acknowledgements}

Research related to this article was funded by the Seventh Framework Programme (CORDIS FP7) of the European Commission (FP7-HEALTH-2011).

\section{Author details}

${ }^{1}$ The EMGO Institute for Health and Care Research, Department of General Practice and Elderly Care Medicine, VU University Medical Center, Amsterdam, The Netherlands. ${ }^{2}$ European Centre on Health of Societies in Transition, London School of Hygiene and Tropical Medicine, London, UK. ${ }^{3}$ Department of Movement and Sport Sciences, Faculty of Medicine and Health Sciences, Ghent University, Ghent, Belgium. ${ }^{4}$ Université Paris 13,
Sorbonne Paris Cité - UREN (Unité de Recherche en Epidémiologie Nutritionnelle), U557 Inserm; U1125 Inra; Cnam, Centre for Research on Human Nutrition lle-de-France (CRNH IdF), Bobigny, France. ${ }^{5}$ Université Pierre et Marie Curie-Paris 6, Dept of Nutrition Pitié-Salpêtrière Hospital (AP-HP), (CRNH IdF), Institute of Cardiometabolism and Nutrition (ICAN), Paris, France. ${ }^{6}$ Research Unit on Nutritional Epidemiology INSERM U557, Paris 13, Human Nutrition Research Center of lle de France and Paris Est University, Lab-Urba Urban Institut of Paris, UPEC, Créteil, France. ${ }^{7}$ The EMGO Institute for Health and Care Research, Department of Epidemiology and Biostatistics, VU University Medical Center, Amsterdam, The Netherlands.

Received: 16 October 2013 Accepted: 11 February 2014

Published: 6 March 2014

\section{References}

1. Finucane MM, Stevens GA, Cowan MJ, Danaei G, Lin JK, Paciorek CJ, Singh GM, Gutierrez HR, Lu Y, Bahalim AN, Farzadfar F, Riley LM, Ezzati M: National, regional, and global trends in body-mass index since 1980: systematic analysis of health examination surveys and epidemiological studies with 960 country-years and $9 \cdot 1$ million participants. Lancet 2011, 377:557-567.

2. Must A, Spadano J, Coakley EH, Field AE, Colditz G, Dietz WH: The disease burden associated with overweight and obesity. JAMA 1999, 282:1523-1529.

3. Comuzzie AG, Allison DB: The search for human obesity genes. Science 1998, 280:1374-1377.

4. Booth KM, Pinkston MM, Poston WS: Obesity and the built environment. J Am Diet Assoc 2005, 105(5 Suppl 1):S110-S117.

5. Swinburn B, Egger G, Raza F: Dissecting obesogenic environments: the development and application of a framework for identifying and prioritizing environmental interventions for obesity. Prev Med 1999, 29(6 Pt 1):563-570.

6. Egger $G$, Swinburn B: An "ecological" approach to the obesity epidemic. Bmj 1997, 315:477-480

7. Papas MA, Alberg AJ, Ewing R, Helzlsouer KJ, Gary TL, Klassen AC: The built environment and obesity. Epidemiol Rev 2007, 29:129-143.

8. Ferdinand A O, Sen B, Rahurkar S, Engler S, Menachemi N: The relationship between built environments and physical activity: a systematic review. Am J Public Health 2012, 102:e7-e13.

9. Feng J, Glass TA, Curriero FC, Stewart WF, Schwartz BS: The built environment and obesity: a systematic review of the epidemiologic evidence. Heal Place 2010, 16:175-190.

10. Durand CP, Andalib M, Dunton GF, Wolch J, Pentz MA: A systematic review of built environment factors related to physical activity and obesity risk: implications for smart growth urban planning. Obes Rev, 12:e173-e182.

11. Sallis JF, Floyd MF, Rodríguez DA, Saelens BE: Role of built environments in physical activity, obesity, and cardiovascular disease. Circulation 2012, 125:729-737.

12. Ding D, Gebel K: Built environment, physical activity, and obesity: what have we learned from reviewing the literature? Heal Place 2012, 18:100-105.

13. Grasser G, Van Dyck D, Titze S, Stronegger W: Objectively measured walkability and active transport and weight-related outcomes in adults: a systematic review. Int J Public Health 2013, 58:615-625.

14. Humpel N, Owen N, Leslie E: Environmental factors associated with adults' participation in physical activity: a review. Am J Prev Med 2002, 22:188-199.

15. Saelens BE, Handy SL: Built environment correlates of walking: a review. Med Sci Sports Exerc 2010, 40:S550-S566.

16. Fleischhacker SE, Evenson KR, Rodriguez DA, Ammerman AS: A systematic review of fast food access studies. Obes Rev 2011, 12:e460-e471.

17. Holsten JE: Obesity and the community food environment: a systematic review. Public Heal Nutr 2009, 12:397-405.

18. Mallen C, Peat G, Croft P: Quality assessment of observational studies is not commonplace in systematic reviews. J Clin Epidemiol 2006, 59:765-769.

19. Lytle LA: Measuring the food environment: state of the science. Am J Prev Med 2009, 36(4 Suppl):S134-S144.

20. Brownson RC, Hoehner CM, Day K, Forsyth A, Sallis JF: Measuring the built environment for physical activity. 2010, 36:S99-S123.

21. Kirtland KA, Porter DE, Addy CL, Al E: Environmental measures of physical activity supports: perception versus reality. Am J Prev Med 2003, 24:323-331. 
22. Kweon BS, Ellis CD, Lee SW, Al E: Large-scale environmental knowledge: investigating the relationship between self-reported and objectivelymeasured physical environments. Environ Behav 2006, 38:72-91.

23. Thomas BH, Ciliska D, Dobbins M, Micucci S: A process for systematically reviewing the literature: Providing the research evidence for public health nursing interventions. Worldviews Evid Based Nurs 2004, 1:176-184

24. Deeks JJ, Dinnes J, D'Amico R, Sowden AJ, Sakarovitch C, Song F, Petticrew M, Altman DG: Evaluating non-randomised intervention studies. Health Technol Assess 2003, 7:iii-x. 1-173.

25. Von Elm E, Altman DG, Egger M, Pocock SJ, Gotzsche PC, Vandenbroucke JP: STROBE initiative: The strengthening the reporting of observational studies in epidemiology (STROBE) statement: guidelines for reporting observational studies. J Clin Epidemio/ 2008, 61:344-349.

26. Stroup DF, Berlin JA, Morton SC, Olkin I, Rennie D, Moher D, Becker BJ, Sipe TA, Thacker SB: Meta-analysis of observational studies in epidemiology. Jama 2000, 283:2008-2012.

27. Moher D, Cook DJ, Eastwood S, Olkin I, Rennie D, Stroup DF: Improving the quality of reports of meta-analyses of randomised controlled trials: the QUOROM statement. Lancet 1999, 354:1896-1900.

28. Ahern M, Brown C, Dukas S: A national study of the association between food environments and county-level health outcomes. J Rural Heal, 27:367-379.

29. Black JL, Macinko J: The changing distribution and determinants of obesity in the neighborhoods of New York City, 2003-2007. Am J Epidemiol 2009, 171:765-775.

30. Black JL, Macinko J, Dixon LB, Fryer GE: Neighborhoods and obesity in New York City. Health Place 2010, 16:489-499.

31. Block JP, Christakis NA, O'Malley AJ, Subramanian SV: Proximity to food establishments and body mass index in the Framingham Heart Study offspring cohort over 30 years. Am J Epidemiol 2011, 174:1108-1114.

32. Bodea TD, Garrow LA, Meyer MD, Ross CL: Socio-demographic and built environment influences on the odds of being overweight or obese: The Atlanta experience. Transp Res Part a-Policy Pract 2009, 43:430-444.

33. Bodor JN, Rice JC, Farley TA, Swalm CM, Rose D: The association between obesity and urban food environments. J Urban Heal 2010, 87:771-781.

34. Brown BB, Yamada I, Smith KR, Zick CD, Kowaleski-Jones L, Fan JX: Mixed land use and walkability: Variations in land use measures and relationships with BMI, overweight, and obesity. Heal Place 2009, 15:1130-1141.

35. Brown BB, Smith KR, Hanson H, Fan JX, Kowaleski-Jones L, Zick CD: Neighborhood design for walking and biking: physical activity and body mass index. Am J Prev Med 2013, 44:231-238.

36. Casagrande SS, Gittelsohn J, Zonderman AB, Evans MK, Gary-Webb TL: Association of walkability with obesity in Baltimore City, Maryland. Am J Public Heal 2011, 101(Suppl):S318-S324.

37. Chen SE, Florax RJ, Snyder SD: Obesity and fast food in urban markets: a new approach using geo-referenced micro data. Heal Econ 2012, 22:835-856.

38. Doyle S, Kelly-schwartz A, Schlossberg M, Stockard J: Active Community Environments and Health: The Relationship of Walkable and Safe Communities to Individual Health. J Am Plan Assoc 2006, 72:19-31.

39. Drewnowski A, Aggarwal A, Hurvitz PM, Monsivais P, Moudon AV: Obesity and supermarket access: proximity or price? Am J Public Heal 2012, 102:e74-e80.

40. Eid J, Overman HG, Puga D, Turner MA: Fat city: Questioning the relationship between urban sprawl and obesity. J Urban Econ 2008, 63:385-404

41. Ewing $R$, Schmid $T$, Killingsworth $R$, Zlot A, Raudenbush S: Relationship between urban sprawl and physical activity, obesity, and morbidity. Am J Heal Promot 2003, 18:47-57.

42. Frank LD, Andresen MA, Schmid TL: Obesity relationships with community design, physical activity, and time spent in cars. Am J Prev Med 2004, 27:87-96.

43. Frank LD, Kavage S: Urban planning and public health: a story of separation and reconnection. J Public Heal Manag Pr 2008, 14:214-220.

44. Frank LD, Saelens BE, Powell KE, Chapman JE: Stepping towards causation: do built environments or neighborhood and travel preferences explain physical activity, driving, and obesity? Soc Sci Med 2007, 65:1898-1914.

45. Frank LD, Sallis JF, Conway TL, Chapman JE, Saelens BE, Bachman W: Many pathways from land use to health - Associations between neighborhood walkability and active transportation, body mass index, and air quality. J Am Plan Assoc 2007, 72:75-87.
46. Frank LD, Sallis JF, Saelens BE, Leary L, Cain K, Conway TL, Hess PM: The development of a walkability index: application to the Neighborhood Quality of Life Study. Br J Sports Med, 44:924-933.

47. Gibson DM: The neighborhood food environment and adult weight status: estimates from longitudinal data. Am J Public Heal 2011, 101:71-78.

48. Gregson J: Poverty, sprawl, and restaurant types influence body mass index of residents in California counties. Public Heal Rep 2011, 126(Supplement 1):141-149.

49. Hattori A, An R, Sturm R: Neighborhood food outlets, diet, and obesity among California adults, 2007 and 2009. Prev Chronic Dis 2013, 10:E35.

50. Hoehner CM, Handy SL, Yan Y, Blair SN, Berrigan D: Association between neighborhood walkability, cardiorespiratory fitness and body-mass index. Soc Sci Med, 73:1707-1716.

51. Hutchinson PL, Nicholas Bodor J, Swalm CM, Rice JC, Rose D: Neighbourhood food environments and obesity in southeast Louisiana. Heal Place 2012, 18:854-860.

52. Inagami S, Cohen DA, Brown AF, Asch SM: Body mass index, neighborhood fast food and restaurant concentration, and car ownership. J Urban Health 2009, 86:683-695.

53. James P, Troped PJ, Hart JE, Joshu CE, Colditz GA, Brownson RC, Ewing R, Laden F: Urban Sprawl, Physical Activity, and Body Mass Index: Nurses' Health Study and Nurses' Health Study II. Am J Public Heal 2012, 103:369-375.

54. Jeffery RW, Baxter J, McGuire M, Linde J: Are fast food restaurants an environmental risk factor for obesity? Int J Behav Nutr Phys Act 2006, 3:2.

55. Jilcott SB, McGuirt JT, Imai S, Evenson KR: Measuring the Retail Food Environment in Rural and Urban North Carolina Counties. J Public Heal Manag Pract, 16:432-440.

56. Keegan TH, Hurley S, Goldberg D, Nelson DO, Reynolds P, Bernstein L, HornRoss PL, Gomez SL: The association between neighborhood characteristics and body size and physical activity in the California teachers study cohort Am J Public Heal 2012, 102:689-697.

57. Lesser LI, Zimmerman FJ, Cohen DA: Outdoor advertising, obesity, and soda consumption: a cross-sectional study. BMC Public Health 2013, 13:20.

58. Lopez R: Urban sprawl and risk for being overweight or obese. Am J Public Heal 2004, 94:1574-1579.

59. Lopez RP: Neighborhood risk factors for obesity. Obes (Silver Spring) 2007, 15:2111-2119.

60. Lovasi GS, Hutson MA, Guerra M, Neckerman KM: Built environments and obesity in disadvantaged populations. Epidemiol Rev 2009, 31:7-20.

61. Lovasi GS, Bader MD, Quinn J, Neckerman K, Weiss C, Rundle A: Body mass index, safety hazards, and neighborhood attractiveness. Am J Prev Med 2012, 43:378-384.

62. McDonald KN, Oakes JM, Forsyth A: Effect of street connectivity and density on adult BMI: results from the Twin Cities Walking Study. J Epidemiol Community Health 2012, 66:636-640.

63. Mehta N, MSc, Chang WW, MD P: Weight status and restaurant availability: a multilevel analysis. Am J Prev Med 2008, 34:127-133.

64. Mobley LR, Root ED, Finkelstein EA, Khavjou O, Farris RP, Will JC: Environment, obesity, and cardiovascular disease risk in low-income women. Am J Prev Med 2006, 30:327-332.

65. Morland K, Diez Roux AV, Wing S: Supermarkets, other food stores, and obesity: the atherosclerosis risk in communities study. Am J Prev Med 2006, 30:333-339.

66. Oka M, Link CL, Kawachi I: Area-based variations in obesity are more than a function of the food and physical activity environment: area-based variations in obesity. J Urban Heal 2012. Epub ahead.

67. Plantinga AJ, Bernell S: The association between urban sprawl and obesity: Is it a two-way street? J Reg Sci 2007, 47:857-879.

68. Plantinga AJ, Bernell S: Can urban planning reduce obesity? The role of self-selection in explaining the link between weight and urban sprawl. Rev Agric Econ 2007, 29:557-563.

69. Rundle A, Diez Roux AV, Free LM, Miller D, Neckerman KM, Weiss CC: The urban built environment and obesity in New York City: a multilevel analysis. Am J Heal Promot 2007, 21(4 Suppl):326-334.

70. Rundle A, Field S, Park Y, Freeman L, Weiss CC, Neckerman K: Personal and neighborhood socioeconomic status and indices of neighborhood walk-ability predict body mass index in New York City. Soc Sci Med 2008, 67:1951-8.

71. Rundle A, Neckerman KM, Freeman L, Lovasi GS, Purciel M, Quinn J, Richards C, Sircar N, Weiss C: Neighborhood food environment and 
walkability predict obesity in New York City. Env Heal Perspect 2009, 117:442-447.

72. Rundle A, Quinn J, Lovasi G, Bader MD, Yousefzadeh P, Weiss C, Neckerman K: Associations between body mass index and park proximity, size, cleanliness and recreational facilities. Am J Heal Promot 2013, 27:262-9.

73. Sallis JF, Saelens BE, Frank LD, Conway TL, Slymen DJ, Cain KL, Chapman JE, Kerr J: Neighborhood built environment and income: examining multiple health outcomes. Soc Sci Med 2009, 68:1285-1293.

74. Samimi A, Mohammadian A, Madanizadeh S: Effects of transportation and built environment on general health and obesity. Transp Res Part D-Transport Environ 2009, 14:67-71.

75. Scott MM, Dubowitz T, Cohen DA: Regional differences in walking frequency and BMI: what role does the built environment play for Blacks and Whites? Heal Place 2009, 15:897-902.

76. Smith KR, Brown BB, Yamada I, Kowaleski-Jones L, Zick CD, Fan JX: Walkability and body mass index density, design, and new diversity measures. Am Prev Med 2008, 35:237-244.

77. Smith KR, Zick CD, Kowaleski-Jones L, Brown BB, Fan JX, Yamada I: Effects of neighborhood walkability on healthy weight: assessing selection and causal influences. Soc Sci Res 2011, 40:1445-1455.

78. Wang MC, Kim S, Gonzalez AA, MacLeod KE, Winkleby MA: Socioeconomic and food-related physical characteristics of the neighbourhood environment are associated with body mass index. J Epidemiol Community Health 2007, 61:491-8.

79. West ST, Shores KA, Mudd LM: Association of Available Parkland, Physical Activity, and Overweight in America's Largest Cities. J Public Heal Manag Pr 2012, 18:423-430.

80. Yamada I, Brown BB, Smith KR, Zick CD, Kowaleski-Jones L, Fan JX: Mixed land use and obesity: an empirical comparison of alternative land use measures and geographic scales. Prof Geogr 2011, 64:157-177.

81. Zhao Z, Kaestner R: Effects of urban sprawl on obesity. J Heal Econ 2010, 29:779-787.

82. Zick CD, Smith KR, Fan JX, Brown BB, Yamada I, Kowaleski-Jones L: Running to the store? The relationship between neighborhood environments and the risk of obesity. Soc Sci Med 2009, 69:1493-1500.

83. Zick CD, Hanson H, Fan JX, Smith KR, Kowaleski-Jones L, Brown BB, Yamada l: Re-visiting the relationship between neighbourhood environment and BMl: an instrumental variables approach to correcting for residential selection bias. Int J Behav Nutr Phys Act 2013, 10:27.

84. Bai H, Wilhelm Stanis SA, Kaczynski AT, Besenyi GM: Perceptions of neighborhood park quality: associations with physical activity and body mass index. Ann Behav Med 2013, 45(Suppl 1):S39-48.

85. Catlin TK, Simoes EJ, Brownson RC: Environmental and policy factors associated with overweight among adults in Missouri. Am J Heal Promot 2003, 17:249-258.

86. Mujahid MS, Diez Roux AV, Shen M, Gowda D, Sanchez B, Shea S, Jacobs DR Jr, Jackson SA: Relation between neighborhood environments and obesity in the Multi-Ethnic Study of Atherosclerosis. Am J Epidemiol 2008, 167:1349-1357.

87. Powell-Wiley TM, Ayers CR, de Lemos JA, Lakoski SG, Vega GL, Grundy S, Das SR, Banks-Richard K, Albert MA: Relationship between perceptions about neighborhood environment and prevalent obesity: data from the Dallas Heart Study. Obesity (Silver Spring) 2013, 21:E14-21.

88. Wilson DK, Ainsworth BE, Bowles H: Body mass index and environmental supports for physical activity among active and inactive residents of a U. S. southeastern county. Heal Psychol 2007, 26:710-717.

89. Tilt $J H$, Unfried TM, Roca B: Using objective and subjective measures of neighborhood greenness and accessible destinations for understanding walking trips and BMI in Seattle. Washington. Am J Heal Promot 2007, 21(4 Suppl):371-379

90. Boehmer TK, Hoehner CM, Deshpande AD, Brennan Ramirez LK, Brownson RC: Perceived and observed neighborhood indicators of obesity among urban adults. Int J Obes 2007, 31:968-977.

91. Rutt CD, Coleman KJ: Examining the relationships among built environment, physical activity, and body mass index in El Paso, TX. Prev Med 2005, 40:831-841.

92. Pendola R, Gen S: BMI, auto use, and the urban environment in San Francisco. Health Place 2007, 13:551-6.

93. Joshu CE, Boehmer TK, Brownson RC, Ewing R: Personal, neighbourhood and urban factors associated with obesity in the United States. J Epidemiol Community Heal 2008, 62:202-208.
94. Berry TR, Spence JC, Blanchard CM, Cutumisu N, Edwards J, Selfridge G: A longitudinal and cross-sectional examination of the relationship between reasons for choosing a neighbourhood, physical activity and body mass index. Int J Behav Nutr Phys Act 2010, 7:57.

95. Spence JC, Cutumisu N, Edwards J, Raine KD, Smoyer-Tomic K: Relation between local food environments and obesity among adults. BMC Public Health 2009, 9:192.

96. Pouliou T, Elliott SJ: Individual and socio-environmental determinants of overweight and obesity in Urban Canada. Heal Place 2010, 16:389-398.

97. Prince SA, Kristjansson EA, Russell K, Billette JM, Sawada MC, Ali A, Tremblay MS, Prud'homme D: Relationships between neighborhoods, physical activity, and obesity: a multilevel analysis of a large Canadian City. Obes (Silver Spring) 2012, 20:2093-100.

98. Prince SA, Kristjansson EA, Russell K, Billette J, Sawada M, Ali A, Tremblay MS, Prud D: A multilevel analysis of neighbourhood built and social environments and adult self-reported physical activity and body mass index in Ottawa. Canada 2011, 7600(July):3953-3978.

99. Brownson RC, Hoehner CM, Day K, Forsyth A, Sallis JF: Measuring the Built Environment for Physical Activity State of the Science. Am J Prev Med 2009, 36:S99-S123.

100. Kestens Y, Lebel A, Chaix B, Clary C, Daniel M, Pampalon R, Theriault M, Subramanian SVP: Association between activity space exposure to food establishments and individual risk of overweight. PLOS One 2012, 7:e41418.

101. Berry TR, Spence JC, Blanchard C, Cutumisu N, Edwards J, Nykiforuk C: Changes in BMl over 6 years: the role of demographic and neighborhood characteristics. Int J Obes (Lond) 2010, 34:1275-83.

102. Garden FL, Jalaludin BB: Impact of urban sprawl on overweight, obesity, and physical activity in Sydney, Australia. J Urban Heal 2008, 86:19-30.

103. Pearce J, Hiscock R, Blakely T, Witten K: A national study of the association between neighbourhood access to fast-food outlets and the diet and weight of local residents. Heal Place 2009, 15:193-197.

104. Richardson EA, Pearce J, Mitchell R, Kingham S: Role of physical activity in the relationship between urban green space and health. Public Health 2013, 127:318-24.

105. Christian H, Giles-Corti B, Knuiman M, Timperio A, Foster S: The influence of the built environment, social environment and health behaviors on body mass index. results from RESIDE. Prev Med (Baltim) 2011, 53:57.

106. Gebel K, Bauman AE, Sugiyama T, Owen N: Mismatch between perceived and objectively assessed neighborhood walkability attributes: prospective relationships with walking and weight gain. Heal Place 2011, 17:519-524.

107. Giles-Corti B, Macintyre S, Clarkson JP, Pikora T, Donovan RJ: Environmental and lifestyle factors associated with overweight and obesity in Perth, Australia. Am J Heal Promot 2003, 18:93-102.

108. Ball K, Lamb K, Travaglini N, Ellaway A: Street connectivity and obesity in Glasgow, Scotland: impact of age, sex and socioeconomic position. Health Place 2012, 18:1307-13.

109. Van Dyck D, Cerin E, Cardon G, Deforche B, Sallis JF, Owen N, de Bourdeaudhuij I: Physical activity as a mediator of the associations between neighborhood walkability and adiposity in Belgian adults. Heal Place 2010, 16:952-960.

110. Santana $P$, Santos $R$, Nogueira $H$ : The link between local environment and obesity: a multilevel analysis in the Lisbon Metropolitan Area, Portugal. Soc Sci Med 2009, 68:601-609.

111. Ellaway A, Macintyre S, Bonnefoy X: Graffiti, greenery, and obesity in adults: secondary analysis of European cross sectional survey. Bmj 2005, 331:611-612.

112. Macdonald L, Ellaway A, Ball K, Macintyre $S$ : Is proximity to a food retail store associated with diet and BMI in Glasgow, Scotland? BMC Public Health 2011, 11:464.

113. Leal C, Bean K, Chaix B: Multicollinearity in associations between multiple environmental features and body weight and abdominal fat: using matching techniques to assess whether the associations are Separab. Am J Epidemiol 2012, 173:S47.

114. Burgoine T, Alvanides S, Lake AA: Assessing the obesogenic environment of North East England. Heal Place 2011, 17:738-747.

115. Coombes $E$, Jones AP, Hillsdon M: The relationship of physical activity and overweight to objectively measured green space accessibility and use. Soc Sci Med 2010, 70:816-822.

116. Cummins S, Fagg J: Does greener mean thinner? Associations between neighbourhood greenspace and weight status among adults in England. Int J Obes 2012, 36:1108-1113. 
117. Poortinga W: Perceptions of the environment, physical activity, and obesity. Soc Sci Med 2006, 63:2835-2846.

118. Toftager M, Ekholm O, Schipperijn J, Stigsdotter U, Bentsen P, Gronbaek M, Randrup TB, Kamper-Jorgensen F: Distance to green space and physical activity: a Danish national representative survey. J Phys Act Heal 2011, 8:741-749.

119. Nielsen TS, Hansen KB: Do green areas affect health? Results from a Danish survey on the use of green areas and health indicators. Health Place 2007, 13:839-850

120. Saelens BE, Sallis JF, Black JB, Chen D: Neighborhood-based differences in physical activity: an environment scale evaluation. Am J Public Health 2003, 93:1552-8.

121. Spittaels H, Verloigne M, Gidlow C, Gloanec J, Titze S, Foster C, Oppert J-M, Rutter H, Oja P, Sjöström M, De Bourdeaudhuij I: Measuring physical activity-related environmental factors: reliability and predictive validity of the European environmental questionnaire ALPHA. Int J Behav Nutr Phys Act 2010, 7:48.

122. Li F, Fisher KJ, Brownson RC, Bosworth M: Multilevel modelling of built environment characteristics related to neighbourhood walking activity in older adults. Community Health J Epidemiol 2005, 59:558-64.

123. Alexander A, Bergman P, Hagstromer M, Sjostrom M: IPAQ environmental module; reliability testing. J Public Heal 2006, 14:76-80.

124. Kremers SPJ, de Bruijn GJ, Visscher TLS, van Mechelen W, de Vries NK, Brug $J$ : Environmental influences on energy balance-related behaviors: a dual-process view. Int J Behav Nutr Phys Act 2006, 3.

125. Tak NI, te Velde SJ, Kamphuis CB, Ball K, Crawford D, Brug J, van Lenthe FJ: Associations between neighbourhood and household environmental variables and fruit consumption: exploration of mediation by individual cognitions and habit strength in the GLOBE study. Public Health Nutr 2013, 16:505-514.

126. Lakerveld J, Brug J, Bot S, Teixeira P, Rutter H, Woodward E, Samdal O, Stockley L, De Bourdeaudhuij I, van Assema P, Robertson A, Lobstein T, Oppert JM, Adany R, Nijpels G: Sustainable prevention of obesity through integrated strategies: The SPOTLIGHT project's conceptual framework and design. BMC Public Health 2012, 12:793.

127. Lopez R, Hynes HP: Obesity, physical activity, and the urban environment: Public health research needs. Environ Heal 2006, 5:25.

128. Lee BA, Reardon SF, Firebaugh G, Farrell CR, Matthews SA, O'Sullivan D: Beyond the census tract: patterns and determinants of racial segregation at multiple geographic scales. Am Sociol Rev 2008, 73:766-791

doi:10.1186/1471-2458-14-233

Cite this article as: Mackenbach et al: Obesogenic environments: a systematic review of the association between the physical environment and adult weight status, the SPOTLIGHT project. BMC Public Health 2014 14:233.

\section{Submit your next manuscript to BioMed Central and take full advantage of:}

- Convenient online submission

- Thorough peer review

- No space constraints or color figure charges

- Immediate publication on acceptance

- Inclusion in PubMed, CAS, Scopus and Google Scholar

- Research which is freely available for redistribution 